\title{
Lapurdum
}

Euskal ikerketen aldizkaria | Revue d'études basques |

Revista de estudios vascos | Basque studies review

$13 \mid 2009$

Numéro XIII

\section{Disparition de la racine verbale et généralisation de l'emploi du participe : le cas de l'ancienne périphrase de perfectif en diachronie}

\section{Céline Mounole}

\section{(2) OpenEdition}

Journals

\section{Édition électronique}

URL : http://journals.openedition.org/lapurdum/2143

DOI : 10.4000/lapurdum.2143

ISSN : 1965-0655

Éditeur

IKER

\section{Édition imprimée}

Date de publication : 1 février 2009

Pagination : $277-287$

ISBN : 978-2-86781-409-X

ISSN : $1273-3830$

\section{Référence électronique}

Céline Mounole, « Disparition de la racine verbale et généralisation de l'emploi du participe : le cas de l'ancienne périphrase de perfectif en diachronie », Lapurdum [En ligne], 13 | 2009, mis en ligne le 15 avril 2013, consulté le 19 avril 2019. URL : http://journals.openedition.org/lapurdum/2143 ; DOI : 10.4000/lapurdum.2143 


\title{
Disparition de la racine verbale et généralisation de l'emploi du participe: le cas de l'ancienne périphrase de perfectif en diachronie
}

\author{
Céline MOUNOLE \\ UPV/EHU - Bordeaux 3
}

\section{Résumé:}

Au cours de l'histoire des dialectes occidentaux et centraux, les périphrases composées de la racine verbale / ou du participe et des auxiliaires *edin, *ezan, egin ont subi un changement dans la forme de leur verbe principal. Les bascologues qui ont étudié cette modification (Azkue 1924, 1935, Lafon 1943, Lakarra 1985a) l'ont présentée comme la généralisation de l'emploi du participe au sein de ces trois périphrases. Cependant, les données des textes des 15, 16, 17 et $18^{\text {ème }}$ siècles semblent montrer des tendances différentes selon les dialectes et, surtout, selon les auxiliaires. Les périphrases à auxiliaires * edin et *ezan ont généralisé l'emploi du participe à des époques différentes, et celle à auxiliaire egin, elle, semble toujours avoir principalement employé le participe comme verbe principal. Ces données nous font donc état de trois périphrases grammaticalisées à partir de deux structures différentes: (i) racine verbale + auxiliaire (périphrases à auxiliaires *edin et *ezan), (ii) participe + auxiliaire (périphrase à auxiliaire egin).

\section{Abstract:}

In the history of the occidental and central dialects of Basque, the verbal periphrases composed by the verbal stem / or the participle and the auxiliaries *edin, *ezan, egin have undergone a change in the form of their principal verb. Until now, the bascologists have presented this modification as the generalization of the use of the participle within these three periphrases. Anyway, the $15^{\text {th }}, 16^{\text {th }}$, $17^{\text {th }}$ and $18^{\text {th }}$ century texts' data seem to show different tendencies depending on the auxiliaries and the periods. The periphrasis based on the auxiliaries *edin and *ezan have generalized the use of the participle at different times, and the egin auxiliary based one seems to have always principally employed the participle as its lexical verb. Thus, these data give us good evidence for proposing that 
these three periphrases appeared and grammaticalized on the base of two different structures: (i) verbal stem + auxiliary (periphrases with the auxiliaries *edin and *ezan), (ii) participle + auxiliary (periphrasis with the auxiliary egin).

Mots-clés : Evolution diachronique, racine verbale, participe, analogie, construction périphrastique, topicalisation

Keywords : Diachronic evolution, verbal stem, participle, analogy, periphrastic construction, topicalisation.

\section{Introduction}

La langue basque du $16^{\text {ème }}$ siècle possède peu de verbes fléchis (une soixantaine) et de nombreuses périphrases. Comme nous avons déjà eu l'occasion de l'exprimer (Mounole 2006), nous pensons que les périphrases composées de la racine verbale ou du participe et des auxiliaires intransitif *edin, et transitifs *ezan, egin sont les plus anciennes du système. De nos jours limitées aux subjonctif, impératif, potentiel, et conditionnel, elles présentent des emplois indicatifs dans les textes les plus anciens.

$\mathrm{Au} 16^{\mathrm{e} m e}$ siècle, tous les dialectes emploient les périphrases à auxiliaires *edin (intransitif) et *ezan (transitif). Les dialectes occidentaux et centraux possèdent en plus une deuxième périphrase transitive composée du participe ou de la racine verbale et de l'auxiliaire egin « faire ». Cette dernière remplaça peu à peu la périphrase à auxiliaire *ezan qui, dès le $18^{\text {ème }}$ siècle, disparut totalement des dialectes occidentaux (voir Mounole 2008).

Dans les textes les plus anciens, - comme de nos jours, d'ailleurs - les dialectes orientaux ne prennent que la racine verbale comme verbe principal de ces périphrases, alors que les dialectes occidentaux et centraux la remplacent parfois par le participe. Aujourd'hui, la racine verbale a disparu des dialectes occidentaux et centraux, ${ }^{1}$ et le participe est employé comme seul verbe principal possible de ces périphrases.

C'est précisément ce phénomène de remplacement de la racine verbale par le participe que nous allons étudier dans cet article. A travers l'étude systématique des textes des 15, 16, 17, et $18^{\text {ème }}$ siècles, nous allons tenter de préciser les directions et chronologies des changements.

Après avoir brièvement présenté le corpus étudié (2) nous allons présenter les données issues de l'examen des textes (3). Nous terminerons en proposant quelques pistes pour appréhender l'évolution formelle de ces périphrases et tenterons de nouvelles hypothèses concernant leurs création et chronologie (4 et 5).

\section{Corpus}

Afin d'offrir une description précise de cette évolution, nous avons étudié de façon systématique les textes occidentaux et centraux des $15^{\text {ème }}, 16^{\text {ème }}, 17^{\text {ème }}$ et $18^{\text {ème }}$ siècles. Nous avons relevé tous les exemples de ces périphrases, et les avons classés selon leurs valeurs. Voici le corpus étudié :

1. En biscayen moderne on ne rencontre la racine verbale que dans quelques expressions fossilisées du type geldi bedi ondo, ken ortik, or konpon Mari Anton, alda, aida adi (Azkue 1924, 1935). 


\subsection{Biscayen}

(i) Avant le 16 ${ }^{\text {ème }}$ siècle: Martin Ibañez (TAV 3.2.4, 74.o), Complainte de Milia Lastur (TAV 3.1.5, 75-79), Abendaño (TAV 3.1.6, 79-81), Incendie de Mondragon (TAV 3.1.7., 81-88), $3^{\text {ème }}$ ordre de Saint François (TAV 3.2.2, 142-144), Obsèques de Henao-Bizkai, Garibai 1571 (Urquijo 1919), Refranes y Sentencias 1596 (Lakarra 1995).

(ii) $16^{\text {ème }}$ siècle: Lettre de Zumarraga 1537 (Contr. 5.2.1, 231-236)

(iii) 17 $7^{\text {ème }}$ siècle: Chant d'amour Veguiacaz 1609 (TAV 3.1.22, 122-123), Poésie d'amour Catigatu (Contri. 5.1.4., 222.o), Cadre de Mendieta 1609 (Contri. 5.2.7., 248-250), Viva Jesus (Mitxelena 1954), Chant de Egia 1650 (Urgell 1996), Mikoleta 1653, Kapanaga 1656, Doctrine d'Amileta 1680 (Arejita \& Alberdi 1985), Bertso Bizkaitarrak 1688 (Lakarra 1984), Bulda (Lakarra 1985), Zubia-Lezamiz 1691 (TAV 138-9; TAV 162 ; TAV 167-168 ; Contr. 5.2.11, 280-289)

(iv) 18 ème siècle: Pièce de Noël (Lakarra 1983), Arrêté de Mondragon 1705 (Ozaeta 1992).

\subsection{Alavais}

(i) 16 $6^{\text {eme }}$ siècle: Betolatza 1596 (Mitxelena 1955), Landuccio 1562 (Mitxelena), Lazarraga c. 1564 (Edition électronique d' I. Landa)

(ii) $17^{\text {ème }}$ siècle: Portal 1610 (TAV 3.1.21, 118-122)

(iii) 18 ${ }^{\text {ème }}$ siècle: Gamiz (Goikoetxea 1998), Albeniz 1778 (Altuna 1995, 1998)

\subsection{Guipuzcoan}

(i) $16^{\mathrm{eme}}$ siècle: Psaume Miserere (Akesolo 1982)

(ii) $17^{\text {mè }}$ siècle: Vers satiriques de Tolosa (TAV 3.1.25 127-131), Proverbes d' Isasti (TAV 3.3.3., 176-183), Ave Maria (TAV 3.2.10 162), Salve d'Isasti (TAV 3.2.12), Lettres d'Azpeitia (Contr. 5.2.9., 258-262), Sermon des Franciscains contre la sorcellerie 1648 (TAV 3.2.13, 165167), Vers de la Vierge d'Itziar (Contr. Note bas de page $n^{\circ} 1,210-211$ ), Lettres de Lardizabal (Contr. 5.2.9., 263.o.), Chant de Bretagne (Contr. 5.1.1., 211-216)

(iii) 18 ème siècle: Otxoa Arin 1713, Irazusta 1739, Doctrine de Zegama 1741 (Ondarra 1984), Kardaberatz 1744 (Hidalgo, s.d.), Dialogues de Goierri (Murugarren 1984), Doctrine d'Oikia 1759 (Lakarra 1987)

\section{Résultats de l'étude du corpus}

\subsection{Généralités}

Nous présentons ci-dessous, par auxiliaire, et par dialecte, les données issues de notre étude du corpus. Cela nous permettra d'observer les chronologies et directions des changements.

Rappelons rapidement que le participe basque est dérivé de la racine verbale par adjonction des suffixes participiaux -i ou -tu : etor > etorri, ekar > ekarri, har > hartu, sar > sartu. D'autres verbes ne prennent aucune marque participiale, et par conséquent, comportent une seule et même forme en guise de participe et de racine verbale. Il s'agit des verbes en - n, -o, -gi, -ki (edan, jaso, idoki), des emprunts du germanique (gorde, bota), et des verbes d'origine adjectivale (hil, bete).

Notre objectif étant d'étudier le remplacement de la racine verbale par le participe, nous distinguons ces divers types de participe dans nos tableaux: (i) ceux à suffixe participial -i (de type etorri, ekarri), (ii) ceux à suffixe participial -tu (de type hartu, sartu), (iii) ceux qui ne portent aucune marque participiale. Ainsi, la racine verbale comporte les entrées - $i$ et - tu, et le participe les entrées + $i$ et $+t u$. Les participes ne portant aucune marque participiale sont inclus dans la dernière colonne, sous la dénomination non marqués (n.m.). 
3.2. Structure à auxiliaire*edin «devenir»

\begin{tabular}{|l|rrrrr|}
\hline \multirow{2}{*}{ Biscayen } & \multicolumn{5}{|c|}{${ }^{*}$ edin } \\
& $-i$ & $-t u$ & $+i$ & $+t u$ & \\
\hline $15^{\text {ème }}$ siècle & - & 25 & 6 & 4 & 12 \\
\hline $16^{\text {ème }}$ siècle & - & 4 & - & 1 & 1 \\
\hline $17^{\text {ème }}$ siècle & - & 16 & 19 & 47 & 44 \\
\hline $18^{\text {ème }}$ siècle & - & 17 & 5 & 15 & 12 \\
\hline
\end{tabular}

\begin{tabular}{|l|ccccc|}
\hline \multirow{2}{*}{ Alavais } & \multicolumn{5}{|c|}{${ }^{*}$ edin } \\
\hline $16^{\text {ème }}$ siècle & $-i$ & $-t u$ & $+i$ & $+t u$ & \\
\hline $17^{\text {ème }}$ siècle & - & - & - & - & - \\
\hline $18^{\text {ème }}$ siècle & - & - & 2 & 3 & 5 \\
\hline
\end{tabular}

\begin{tabular}{|l|llllll|}
\hline & \multicolumn{5}{|c|}{${ }^{*}$ edin } \\
Guipuzcoan & $\begin{array}{l}\text { racine } \\
-i\end{array}$ & $-t u$ & $+i$ & participe & n.m. \\
\hline $16^{\text {ème }}$ siècle & - & - & - & 1 & 2 \\
\hline $17^{\text {ème }}$ siècle & - & 3 & - & 1 & 2 \\
\hline $18^{\text {ème }}$ siècle & - & 34 & 13 & 27 & 80 \\
\hline
\end{tabular}

$1^{\text {er }}$ groupe de tableaux: Compétition entre la racine verbale et le participe dans la périphrase à auxiliaire *edin

\subsubsection{Textes des $15^{\text {ème }}$ et $16^{\text {ème }}$ siècles}

Dans les textes du $15^{\text {ème }}$ siècle, la racine verbale est majoritaire avec l'auxiliaire intransitif *edin. Si l'on compare les formes dont le participe est en -tu, on observe que dans $86,2 \%$ des cas c'est la racine verbale qui est employée.

Au $16^{\text {ème }}$ siècle, elle est toujours majoritaire en biscayen et en alavais (60\% des cas en alavais). Cependant, d'un texte à l'autre on observe des différences non négligeables: en alavais, la racine verbale est très courante dans le texte de Lazarraga (39 racines verbales vs 23 participes) alors que Betolatza et Landuccio, eux, ne l'emploient jamais. De plus, des variations sont manifestes selon les valeurs prises par la périphrase : dans le texte de Lazarraga de la racine verbale est majoritaire à l'impératif ( 25 racines verbales vs 4 participes), alors que dans tous les autres emplois c'est le participe qui est préféré (aoriste, potentiel).

Le seul texte guipuzcoan du $16^{\text {ème }}$ siècle (Psaume Miserere), n'offre pas de données nous permettant de tirer de conclusion.

\subsubsection{Textes du $17^{\text {ème }}$ siècle}

Dans les textes biscayens du $17^{\text {ème }}$ siècle, le participe devient largement majoritaire avec l'auxiliaire *edin. Si l'on compare les formes dont le participe est en -tu, on constate qu'il est employé dans $74,6 \%$ des cas.

Néanmoins, en guipuzcoan la racine verbale est toujours préférée dans cette structure : 3 racines verbales contre 1 participe.

\subsubsection{Textes du $18^{\text {ème }}$ siècle}

Dès le siècle suivant, la racine verbale a totalement disparu de la majorité des parlers biscayens. Dans les textes d'Arzadun (1731) et Urkizu (1737) de Durango, et dans l'arrêté de Mondragon (1705) la périphrase à auxiliaire *edin apparaît toujours avec le participe. Le texte de Barrutia 
constitue une exception à cette tendance biscayenne, puisque la racine verbale y est employée comme verbe principal de la structure intransitive dans $58,6 \%$ des cas (formes en -tu comparées).

En alavais, l'auxiliaire *edin est toujours employé avec le participe.

En ce qui concerne le guipuzcoan, le tableau nous apprend qu'au $18^{\text {ème }}$ siècle la racine verbale reste majoritaire dans cette périphrase. Cependant, les données de chaque texte ne concordent pas forcément avec ces données générales. On observe, en effet, trois tendances différentes parmi les cinq textes du $18^{\text {ème }}$ siècle:

(i) Certains emploient principalement le participe: Otxoa Arin, 17 participes contre 2 racines verbales;

(ii) D'autres emploient principalement la racine verbale malgré une avancée du participe qui n'est pas négligeable: Irazusta: 6 racines verbales contre 3 participes, Kardaberatz: 7 racines verbales contre 3 participes;

(iii) D'autres emploient seulement la racine verbale : Dialogues de Goierri : 4 racines verbales contre 0 participe, doctrine d'Oikia : 11 racines verbales contre 1 participe.

\subsubsection{Résumé}

On observe deux tendances différentes dans le remplacement de la racine verbale par le participe dans la structure à auxiliaire intransitif *edin: (i) celle du biscayen et de l'alavais, et (ii) celle du guipuzcoan.

En biscayen et en alavais, la racine verbale est largement majoritaire aux $15^{\text {ème }}$ et $16^{\text {ème }}$ siècles. $\mathrm{Au} 17^{\mathrm{ème}}$ siècle le participe est plus fréquemment employé que la racine verbale et dès le $18^{\text {ème }}$ siècle, il devient le seul verbe principal possible de la périphrase à auxiliaire *edin - mis à part le texte de Barrutia, qui demeure plus archaïsant que ses contemporains, voir Zuazo 2006 -.

En guipuzcoan, la propagation du participe s'est produite bien plus tard, puisque dans la plupart des textes du $18^{\text {ème }}$ siècle, la racine verbale est toujours majoritaire. Otxoa Arin (variété d'Ordizia) est le seul à avoir généralisé l'emploi du participe comme verbe principal de la périphrase à auxiliaire *edin.

\subsection{Structure transitive à auxiliaire *ezan}

\begin{tabular}{|c|c|c|c|c|c|}
\hline \multirow[b]{2}{*}{ Biscayen } & \multicolumn{4}{|c|}{${ }^{*} e d i n$} & \multirow[b]{2}{*}{ n.m. } \\
\hline & & $\begin{array}{l}\text { ne } \\
-t u\end{array}$ & $\begin{array}{r}p \\
+i\end{array}$ & $\begin{array}{l}\text { rticipe } \\
+t u\end{array}$ & \\
\hline $15^{\text {ème }}$ siècle & 2 & 14 & 1 & - & 8 \\
\hline $16^{\text {ème }}$ siècle & - & - & - & - & - \\
\hline $17^{\text {ème }}$ siècle & - & 5 & - & - & - \\
\hline $18^{\text {ème }}$ siècle & - & 10 & - & - & - \\
\hline
\end{tabular}

\begin{tabular}{|l|ccccc|}
\hline & \multicolumn{5}{|c|}{${ }^{*}$ edin } \\
Alavais & $\begin{array}{l}\text { racine } \\
-i\end{array}$ & $-t u$ & $\begin{array}{c}\text { participe } \\
+i\end{array}$ & n.tu. \\
\hline $16^{\text {ème }}$ siècle & - & 30 & - & - & - \\
\hline $17^{\text {ème }}$ siècle & - & - & - & - & - \\
\hline $18^{\text {ème }}$ siècle & - & 1 & - & 2 & - \\
\hline
\end{tabular}

\begin{tabular}{|l|ccccc|}
\hline & \multicolumn{5}{|c|}{${ }^{*} e$ din } \\
Guipuzcoan & $\begin{array}{l}\text { racine } \\
-i\end{array}$ & $-t u$ & $+i$ & participe & n.m. \\
\hline $16^{\text {ème }}$ siècle & - & 12 & - & 1 & 4 \\
\hline $17^{\text {ème }}$ siècle & - & 7 & 3 & 1 & 3 \\
\hline $18^{\text {ème }}$ siècle & - & 107 & 41 & 57 & 100 \\
\hline
\end{tabular}


$2^{\text {ème }}$ groupe de tableaux: Compétition entre la racine verbale et le participe dans la périphrase à auxiliaire *ezan

\subsubsection{Résultats}

En biscayen, la racine verbale est le verbe principal préféré de la périphrase à auxiliaire *ezan à toutes les périodes. En quatre siècles, nous ne trouvons qu'une seule exception à cette tendance : Eznea, guria ta odola errorean atera neban ta ezer yrabaci ez neçan RS 513.

En alavais, ce n'est qu'au $18^{\text {ème }}$ siècle que le participe apparaît dans la périphrase à auxiliaire *ezan, dans le texte d'Albeniz 1778.

En guipuzcoan la racine verbale est la forme courante du verbe principal de la périphrase aux $16^{\text {ème }}$ et $17^{\text {ème }}$ siècles $\left(16^{\text {ème }}\right.$ siècle : 12 racines verbales contre 1 participe ; $17^{\text {ème }}$ siècle : 7 racines verbales contre 1 participe). Au $18^{\text {ème }}$ siècle, on observe deux tendances opposées au sein des parlers guipuzcoans :

(i) certains emploient majoritairement le participe : Otxoa Arin (24 participes contre 3 racines verbales) ;

(ii) d'autres préfèrent la racine verbale : Irazusta (17 racines verbales contre 8 participes), doctrine de Zegama (14 racines verbales contre 8 participes), dialogues de Goierri (6 racines verbales contre 2 participes), doctrine d'Oikiako (36 racines verbales contre 9 participes) et Kardaberatz (31 racines verbales contre 6 participes).

\subsubsection{Résumé}

Dans les trois territoires, la périphrase à auxiliaire *ezan prend généralement la racine verbale comme verbe principal aux $16^{\text {ème }}$ et $17^{\text {ème }}$ siècles.

La propagation du participe dans la périphrase à auxiliaire *ezan ne commence réellement qu'au $18^{\text {ème }}$ siècle, en guipuzcoan et alavais. Pour lors, le biscayen a déjà quasiment totalement remplacé cette structure par celle composée du participe / racine verbale + egin que nous allons étudier maintenant.

\subsection{Structure transitive à auxiliaire egin «faire»}

\begin{tabular}{|l|lllcc|}
\hline \multirow{2}{*}{ Biscayen } & \multicolumn{5}{|c|}{${ }^{*}$ redin } \\
& $-i$ & $-t u$ & $+i$ & $+t u$ & \\
\hline $15^{\text {ème }}$ siècle & - & 2 & 6 & 9 & 12 \\
\hline $16^{\text {eme }}$ siècle & - & - & - & 1 & - \\
\hline $17^{\text {ème }}$ siècle & - & - & 36 & 109 & 125 \\
\hline $18^{\text {ème }}$ siècle & - & - & 2 & 16 & 9 \\
\hline
\end{tabular}

\begin{tabular}{|l|ccccc|}
\hline \multirow{2}{*}{ Alavais } & \multicolumn{5}{|c|}{ *edin } \\
& $\begin{array}{l}\text { racine } \\
-i\end{array}$ & $-t u$ & $\begin{array}{c}\text { participe } \\
+i\end{array}$ & $+t u$ & n.m. \\
\hline $16^{\text {ème }}$ siècle & - & 2 & 17 & 65 & 42 \\
\hline $17^{\text {ème }}$ siècle & - & - & - & - & 2 \\
\hline $18^{\text {ème }}$ siècle & - & 2 & 1 & 14 & 6 \\
\hline
\end{tabular}

\begin{tabular}{|c|c|c|c|c|c|}
\hline \multirow[b]{2}{*}{ Guipuzcoan } & \multicolumn{4}{|c|}{ *edin } & \multirow[b]{2}{*}{ n.m. } \\
\hline & $-i$ & & $\begin{array}{l}\text { par } \\
+i\end{array}$ & $\begin{array}{l}\text { icipe } \\
-t u\end{array}$ & \\
\hline $16^{\text {ème }}$ siècle & & - & - & 2 & - \\
\hline $17^{\text {ème }}$ siècle & - & 3 & 1 & - & 3 \\
\hline $18^{\text {ème }}$ siècle & - & 6 & 14 & 32 & 37 \\
\hline
\end{tabular}


$3^{\text {ème }}$ groupe de tableaux: Compétition entre la racine verbale et le participe dans la périphrase à auxiliaire egin

\subsubsection{Résultats}

Les données de l'auxiliaire egin sont totalement différentes de celles des auxiliaires *edin et *ezan étudiés ci-dessus.

En biscayen, toutes époques confondues, il est employé avec le participe dans 98,5\% des cas. En quatre siècles, on ne le rencontre que deux fois avec la racine verbale comme verbe principal : Oi aldioneri albanegui empara / Barriz enendorque Aramaioco contrara (Abendaño TAV. 3.1.6., 79), Lapurrac ona dau ucoa, uca eyquec gaxtoa RS 203.

On observe exactement la même tendance en alavais. Au $16^{\text {ème }}$ siècle, le participe est employé dans cette périphrase dans $97 \%$ des cas et au $18^{\text {ème }}$ siècle dans $87,5 \%$ des cas.

Dans le court texte guipuzcoan du $16^{\text {ème }}$ siècle, egin n'est employé qu'à deux reprises, les deux fois avec le participe. Au $17^{\text {ème }}$ siècle, c'est la racine verbale qui est préférée ( 3 racines verbales contre aucun participe parmi les formes en -tu). Enfin, au $18^{\text {ème }}$ siècle, le participe est employé dans $84,2 \%$ des cas (soit, 32 participes contre 6 racines verbales parmi les formes en -tu).

\subsubsection{Résumé}

La périphrase à auxiliaire egin montre une évolution contraire à celle des périphrases à auxiliaires *edin et *ezan. Depuis les textes les plus anciens, le participe y est quasiment de règle. Toutes époques et dialectes confondus, il est employé dans 94,3\% des apparitions de la périphrase - formes en -tu comparées -.

Ces données semblent indiquer que le participe est le verbe principal originel de cette périphrase. L'emploi de la racine verbale dans cette structure est sûrement récent et a dû apparaître par analogie avec les périphrases à auxiliaires *edin et *ezan. ${ }^{2}$

\subsection{Conclusion}

Lanalyse des données nous permet de tirer les conclusions suivantes:

a) Le remplacement de la racine verbale par le participe ne s'est produit que dans les périphrases à auxiliaires *edin et *ezan

b) En basque historique, la périphrase à auxiliaire egin est quasiment toujours employée avec le participe comme verbe principal.

c) Le remplacement de la racine verbale par le participe n'est survenu ni en même temps, ni même de la même façon dans les périphrases à auxiliaires *edin et *ezan. Dans les dialectes occidentaux, le participe commence à être employé comme verbe principal de la structure à auxiliaire *edin dès le $16^{\text {ème }}$ siècle, tandis qu'avec l'auxiliaire *ezan la racine verbale est de règle jusqu'au $18^{\text {ème }}$ siècle.

d) Le remplacement de la racine verbale par le participe ne s'est pas produit au même moment

2. Contrairement à l'affirmation de Azkue (1935), la forme « correcte » du verbe principal doit donc être le participe, et non pas la racine verbale : "Por la flexión subjuntiva correspondiente a " para que nos libre » dice siempre Betolaza libradu gagizan y no libra gagizan que sería lo correcto... » (Azkue 1935: 72) 
dans tous les dialectes. En guipuzcoan, la racine verbale s'est perdue bien plus tard qu'en biscayen et en alavais, et en même temps dans les périphrases à auxiliaires *edin et *ezan.

e) Quant à l'expansion géographique du participe, nous proposons une propagation d'ouest en est, de l'ouest de la Biscaye et de l'Alava vers l'est de la Biscaye (Barrutia), puis vers le Guipúzcoa. A l'intérieur du Guipúzcoa aussi nous observons le même mouvement d'ouest en est (doctrine d'Oikia, Irazusta, Kardaberatz contre Otxoa Arin).

f) En ce qui concerne le suffixe participial -i, au $16^{\text {ème }}$ siècle, il n'a déjà plus aucune fonction dans les textes occidentaux et centraux, puisque les verbes à participe en -i ne montrent jamais leur racine verbale. Dans toute l'histoire des dialectes occidentaux (biscayen et alavais) et centraux (guipuzcoan) on ne trouve que deux racine verbales dont le participe prend le suffixe -i : ez azauenac eros aala RS 101, az ezac er[r]oya RS 136. Il est intéressant de souligner que ces deux exemples, tirés du texte Refranes y Sentencias 1596, sont employés la périphrase à auxiliaire *ezan, qui, nous l'avons vu, est très conservatrice en biscayen.

\section{Proposition}

Comme le montre l'étude du corpus, entre les $15^{\text {ème }}$ et $18^{\text {ème }}$ siècles, les périphrases à auxiliaires *edin et *ezan ont connu un changement dans la forme de leur verbe principal dans les dialectes occidentaux et centraux. Nous en concluons qu'elles furent créées avec la racine verbale comme verbe principal ([racine verbale $\left.+{ }^{*} e d i n\right]$ et [racine verbale $\left.+{ }^{*} e z a n\right]$ ), et que ce n'est que plus tard que le participe la remplaça dans certains dialectes. Nous pensons que ces deux périphrases, qui sont communes à tous les dialectes, proviennent du basque unifié ancien (Mitxelena 1981).

D'après les informations que nous fournit le corpus, la périphrase à auxiliaire egin, elle, semble avoir été créée à partir d'une structure de type [participe + egin]. Plus tard, et sûrement par analogie avec les périphrases à auxiliaires *edin et *ezan, la racine verbale y remplaça le participe. Toutefois, ces cas de propagation de la racine verbale restent très marginaux (15 exemples en trois siècles, soit $5,7 \%$ de tous ses emplois).

Notre proposition présente plusieurs avantages : (i) Tout d'abord, elle permet de comprendre la propagation du participe dans les périphrases à auxiliaires *edin et *ezan. Au fur et à mesure que les emplois de la structure [participe + egin] se sont accrus, le participe s'est répandu aux deux autres périphrases par action analogique. (ii) Ensuite, elle explique la distribution géographique de la propagation du participe, puisque cette dernière ne s'est produite que dans les parlers qui possèdent la périphrase à auxiliaire egin. Deux tendances sont cependant notables. Ceux qui emploient principalement la structure à auxiliaire egin comme périphrase transitive ont généralisé l'emploi du participe dans la périphrase à auxiliaire *edin (Otxoa Arin, Gamiz, Kapanaga, VJ, Amileta, Zubia-Lezamiz). Par contre, les variétés qui emploient essentiellement la périphrase transitive à auxiliaire *ezan, elles, continuent à employer la racine verbale comme verbe principal de la périphrase à auxiliaire *edin (Irazusta, Barrutia, dialogues de Goierri, Kardaberatz, doctrines de Zegama eta d'Oikia).

La périphrase à auxiliaire *ezan reste en marge du phénomène d'expansion du participe. Nous l'avons vu, jusque très tard la racine verbale demeure le seul verbe principal possible de cette construction. Dès le $16^{\text {ème }}$ siècle, en biscayen et en alavais, elle occupe une position secondaire dans le système puisqu'elle est déjà fortement remplacée par la périphrase à auxiliaire egin. Il semblerait qu'elle se soit en quelque sorte fossilisée, empêchant ainsi tout changement au sein de sa structure. Cependant, dans les dialectes où cette périphrase est employée de façon productive, et en concurrence avec la périphrase à auxiliaire egin, la racine verbale se voit remplacée par le participe dès le $18^{\text {ème }}$ siècle. 


\section{Création et grammaticalisation de la périphrase [participe + egin]}

Nous pensons que diachroniquement la périphrase [participe + egin] a dû apparaître sous la forme d'une structure bi-clausale. Le participe (1) ou un syntagme nominal dont la tête est un participe (2) devait occuper la fonction de complément du verbe egin:

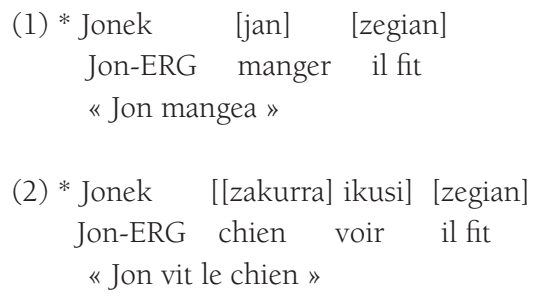

Plus tard, la structure bi-clausale a dû se réanalyser en périphrase verbale, le participe se réanalysant comme verbe principal de la périphrase, et egin comme son auxiliaire (4), (5):

(4) Jonek [jan] [zegian] > Jonek [jan zegian]

(5) Jonek [[zakurra] ikusi] [zegian] > Jonek zakurra [ikusi zegian]

Nous proposons que cette structure fut créée pour topicaliser le verbe participe. Elle dut apporter une opposition marquée vs non marquée au sein des structures transitives :

(6) *Jaungoikoak mundua KREATU zegian (structure marquée)

«Dieu CREA le monde»

vs (7) Jaungoikoak mundua krea zezan (structure non-marquée)

«Dieu créa le monde»

Avec le temps, cette structure a dû devenir plus fréquente et sa valeur initiale (marquée) a dû se perdre. Devenue synonyme de la périphrase [racine verbale $+{ }^{*} e z a n$ ], c'est alors qu'elle a dû commencer à en prendre la place:

\section{Jaungoikoak mundua kreatu zegian (structure non marquée) \\ $=\quad$ Jaungoikoak mundua krea zezan (structure non marquée)}

Pour terminer, les données des langues du monde étayent notre proposition. Il est en effet très courant d'employer le verbe faire comme marque de topicalisation (l'anglais et le wolof en sont témoins, cf. Nougier 2002, Creissels 2006: 114). C'est d'ailleurs ce que l'on trouve dans les dialectes occidentaux du basque moderne, cette fois-ci le verbe egin étant employé périphrastiquement : Peiok etxea saldu dio anaiari (structure non marquée) vs Peiok etxea SALDU egin dio anaiari (structure marquée).

\section{Conclusion}

La propagation du participe au sein des périphrases à auxiliaires *edin et *ezan coïncide avec la 
généralisation de l'emploi de la périphrase transitive [participe + egin]. Au fur et à mesure que cette dernière s'est propagée, le participe a remplacé la racine verbale au sein de la périphrase à auxiliaire *edin en Biscaye, et au sein des périphrases à auxiliaires *edin et *ezan en Guipúzcoa et Alava.

Pour terminer, nous voudrions insister sur le fait que plutôt qu'un simple changement d'auxiliaire, les dialectes occidentaux et centraux ont connu un changement de périphrase [racine verbale $+{ }^{*}$ ezan $]>$ [participe + egin] .

Cette recherche ne fait qu'ajouter une périphrase de plus - [participe + egin] - à la longue liste des périphrases verbales dont dispose la langue basque.

\section{Bibliographie}

Agud, M., \& Mitxelena, K., [1958], 1998, Dictionarium linguae cantabricae (1562), Araberaren lekukoak, H. Knörr \& K. Zuazo (éds.), Vitoria-Gasteiz : Euskal Azterlan Bilduma, 201-334.

Akesolo, L. 1982, «Amaseigarren mendeko euskerazko Miserere bat», Karmel 166, 37-47.

Altuna, F., 1995, «Acto contriciocoa eriotzaco orduracò: Garcia de Albeniz araiarraren araberazko eskuizkribua (1778), ASJU XXXIX: 1, 83-132.

1998, « Garcia de Albeniz araiarraren eskuizkribua», Araberaren lekukoak, H. Knörr \& K. Zuazo (arg.), Vitoria-Gasteiz : Euskal Azterlan Bilduma, 107-123.

Arejita, A. \& Alberdi M., 1985, «Amiletaren dotrinea (XVII. mendea)», Euskeraren Iker- Atalak 3, 7-68.

Arriolabengoa, J., 1996, « Erdi Aroko Kanta ezezagunak Ibargüen-Cachopin kronikan 1620)», ASJU, XXX-71-98.

Azkue, R.M., 1925-27, Morfología vasca, Euskera IV-VI.

—.1932, Verbo guipuzcoano: apéndice de la Morfología vasca, Bilbao: Editorial Vasca.

—.1935, "Evolución de la lengua vasca", Euskera XVI, 1-2, 57-120.

Beriain, J., de, 1621, Tratado de como se ha de oyr missa: escrito en romance, y bascuence, lenguages de este Obispado de Pamplona, Iruñea-Pamplona.

Bonaparte, L.L., 1869, Le verbe basque en tableaux accompagné de notes grammaticales, selon les huits dialectes de l'euskara..., Londres: Strangeways \& Walden.

Creissels, D., 2006, Syntaxe générale: une introduction typologique, Paris: Lavoisier.

Etxaide, J., 1984, “-(e)za aditzerroa datiboko aditz-jokoetan autoreetan zehar”, Euskera XXIX, 601-730.

Goikoetxea, J., 1998, “Olerkiak”, Araberaren lekukoak, H. Knörr \& K. Zuazo (éds.), Vitoria-Gasteiz : Euskal Azterlan Bilduma, 511-516.

Irazusta 1739, Doctrina Christiana eguinzuana erdaraz: Aita Gaspar Astete Jesuitac. Ipinidu eusqueraz D. Juan de Irazuzta, Erretore Hernanialdecoac...

Irigoien, A., 1978, «Canción antigua de amor vizcaína», FLV 30, 453-459.

Kapanaga, M. Otxoa de, 1656 : Exposición breve de la doctrina christiana compuesta por el P. M. Geronimo de Ripalda de la compañia de Iesus.

Kardaberaz, A., 1744, Cristavaren bicitza, (Edition de B. Hidalgo).

Lafon, R., 1943, Le système du verbe basque au XVIème siècle. Bordeaux: Delmas.

—.1999, Vasconiana Iker 11. Bilbao: Académie de la langue basque Euskaltzaindia. 
Lakarra, J., 1983, «Acto para la Nochebuena, Texto y traducción», in ZZEE, Gabonetako ikuskizuna, Gasteiz, 75-126.

—. 1984, «Bertso Bizkaitarrak (1688), ASJU XVIII-2 (1984), 89-184.

—. 1985a, «-tu vs Ø Bizkaiera Zaharrean», Euskeraren Iker Atalak 3, 282-292.

—. 1985b «XVII. mendeko bulda bat bizkaieraz », in J. L. Melena (éd.), Symbolae L. Mitxelena, Gasteiz, 1045-1054.

—. 1986, «Bizkaiera zaharra euskalkien artean », ASJU 20: 3, 639-682.

—. 1987, «Oikiako dotrina (1759)», ASJU XXI-2, 515-564.

—. 1996, Refranes y Sentencias (1596): Ikerketak eta edizioa, Bilbao: Académie de la langue basque Euskaltzaindia.

Lazarraga, J., Perez de c. 1664, Eskuizkribua, Edition électronique d'Iñigo Landa.

Mitxelena, K., 1954, «Textos vascos antiguos. Un catecismo vizcaíno del siglo XVII», BAP 10, 85-95.

—.1955, «La doctrina christiana de Betolaza», BAP 11, 83-100.

—. [1964], 1990, Textos arcaicos vascos, ASJU XI, Donostia-San Sebastian, 5-201.

Mounole, C., 2006, "Quelques remarques à propos de l'histoire des périphrases basques". Studies on Basque and Historical Linguistics in honor to R. L. Trask -Ikerketak Euskalaritzaz eta Hizkuntzalaritza Historikoaz. Larry Trasken Oroitzapenetan, J. A. Lakarra, J. I. Hualde (éds.), Suppléments d'ASJU 40. 1/2, 725-740.

Murugarren, L., 1984, «Una plática del Goyerri», ASJU XVIII- 2, 185-197.

Nouguier, S., 2002, Relations entre fonctions syntactiques et fonctions sémantiques en wolof, Thèse doctorale : Université Lumière Lyon 2.

Ondarra, F., 1984, "Zegamako Doctrina Christiana (1741), ASJU 18:2, 3-62.

Otxoa Arin, J., de 1713 : Doctrina Christianaren explicacioa Villa Franca Guipuzcoaco onetan euscaraz itceguitendan moduan.

Ozaeta, A., 1992, «Arrasateko testu zahar bi», ASJU XXVI-3, 793-800.

Sarasola, I., [1983] 1990, «Contribución al estudio y edición de textos antiguos vascos», ASJU XI, Donostia- San Sebastian, 205-351.

Satrustegi, J.M., 1987, Euskal testu zaharrak, Euskararen lekukoak 13, Iruñea-Pamplona: Académie de la langue basque Euskaltzaindia.

Trask, R. L., 1997, The history of Basque. Londres, Routledge.

Urgell, B., 1986, «Egiaren kantaz : I. testua eta iruzkinak», ASJU XX-1, 75-149.

Urkijo, J., de, 1919, «Los refranes de Garibay», Donostia: Martin, Mena \& Comp.

Zabala, J.M., 1848, El verbo regular vascongado del dialecto vizcaíno, Donostia.

Zuazo, K., 1998a, «Betolatzaren hizkeraz», Araberaren lekukoak, H. Knörr \& K. Zuazo (arg.), Gasteiz: Euskal Azterlan Bilduma, 71-86.

—. 1998b, «Arabera», Araberaren lekukoak, H. Knörr \& K. Zuazo (arg.), Gasteiz: Euskal Azterlan Bilduma, 125-189.

—. 2006, «Deba ibarreko euskara zaharra», Studies on Basque and Historical Linguistics in honor to R. L. Trask -Ikerketak Euskalaritzaz eta Hizkuntzalaritza Historikoaz. Larry Trasken Oroitzapenetan, J. A. Lakarra, J. I. Hualde (arg.), ASJU-ren gehigarriak 40. 1/2, 1005-1029. 